\title{
DC conductivity and Seebeck coefficient of nonstoichiometric MgCuZn ferrites
}

\author{
W. Madhuri ${ }^{1,2, *}$, S. Roopas Kiran $^{1}$, M. Penchal Reddy ${ }^{3}$, N. Ramamanohar Reddy ${ }^{4}$, \\ K.V. SIVA KUMAR ${ }^{5}$ \\ ${ }^{1}$ School of Advanced Sciences, VIT University, Vellore, 632 014, India \\ ${ }^{2}$ IFW, Leibniz Institute for Solid State and Materials Research, Technische Universität Dresden, 01069 Dresden, Germany \\ ${ }^{3}$ Center for Advanced Materials, Qatar University, Doha 2713, Qatar \\ ${ }^{4}$ Department of Materials Science \&Nanotechnology, Yogi Vemana University, Kadapa, 516 227, India \\ ${ }^{5}$ Ceramic Composites Materials Laboratory, Sri Krishnadevaraya University, Anantapur 515 055, India
}

\begin{abstract}
Nonstoichiometric series of $\mathrm{Mg}_{0.5-\mathrm{x}} \mathrm{Cu}_{\mathrm{x}} \mathrm{Zn}_{0.5} \mathrm{Fe}_{1.9} \mathrm{O}_{4-\delta}$ where $\mathrm{x}=0.0,0.1,0.15,0.2$ and 0.25 has been synthesized by conventional solid state reaction route. The single phase spinel structure of the double sintered ferrites was confirmed by $\mathrm{X}$-ray diffraction patterns (XRD). The ferrite series was studied in terms of DC electrical conductivity and thermoelectric power in the temperature ranging from room temperature to $300^{\circ} \mathrm{C}$ and $400{ }^{\circ} \mathrm{C}$, respectively. It was observed that DC electrical conductivity and Seebeck coefficient $\alpha$ decreased with the increase in $\mathrm{x}$. DC electrical conductivity was found to decrease by about 4 orders. All the compositions showed a negative Seebeck coefficient exhibiting n-type semiconducting nature. From the above experimental results, activation energy and mobility of all the samples were estimated. Small polaron hopping conduction mechanism was suggested for the series of ferrites. Owing to their low conductivity the nonstoichiometric $\mathrm{MgCuZn}$ ferrites are the best materials for transformer core and high definition television deflection yokes.
\end{abstract}

Keywords: ferrites; conduction mechanism; Seebeck coefficient; mobility

(C) Wroclaw University of Science and Technology.

\section{Introduction}

Properties of spinels depend on their composition, method of preparation and sintering temperature [1]. Simplicity of the cubic spinel structure has attracted many researchers around the world to explore multi-facet functionality and applications of ferrites. Ferrites occupy an important place in almost every industry branch, from electronics and communications to medical industry, military to space technology [2]. Their structural simplicity allows easy tailoring of materials with desired electrical, thermal, optical and magnetic properties. $\mathrm{MgCuZn}$ ferrites are known for their high environmental stability, low magnetocrystalline anisotropy, low cost and easy handling when compared to their counterpart $\mathrm{NiCuZn}$ ferrites [3-11]. DC electrical resistivity and

\footnotetext{
*E-mail: madhuriw12@gmail.com
}

thermoelectric power of $\mathrm{ZnMgNd}$, NiCuMgZn, NiZnMgCo, MgZn, MgTi and Mg-Fe-O [12-17] spinel systems have been reported by several researchers. However, similar studies on $\mathrm{MgCuZn}$ ferrite system are not available in literature. Slight deficiency of iron in the $\mathrm{MgCuZn}$ ferrites prevents $\mathrm{Fe}^{2+}$ ion formation and thereby decreases the conductivity of ferrites. The present study is aimed at investigating DC electrical resistivity and Seebeck coefficient in a series of $\mathrm{Mg}_{0.5-\mathrm{x}} \mathrm{Cu}_{\mathrm{x}} \mathrm{Zn}_{0.5} \mathrm{Fe}_{1.9} \mathrm{O}_{4-\delta}(\mathrm{x}=0,0.1,0.15,0.2$ and 0.25 ) ferrites for probable applications as core materials of transformer and high definition TV deflection yokes.

\section{Experimental}

Reagent grade $\mathrm{MgO}, \mathrm{CuO}, \mathrm{ZnO}$ and $\mathrm{Fe}_{2} \mathrm{O}_{3}$ were taken in stoichiometric proportions and mixed in an agate mortar for 8 hours. The mixed oxide 
powder was pre-sintered at $800{ }^{\circ} \mathrm{C}$ for 4 hours. The samples were again ground for 10 hours and then sieved for uniform particle size. So obtained green powder was pressed into a pellet of $10 \mathrm{~mm}$ diameter and $2 \mathrm{~mm}$ height with PVA as a binder, using a uniaxial press. The green pellet was annealed at $1150{ }^{\circ} \mathrm{C}$ for 4 hours in a programmable furnace. The dense ferrites were polished to obtain a smooth surface and painted on either side with a silver paste for a good electrical contact. These samples were used for DC electrical conductivity and thermoelectric power studies. DC resistivity was measured by two probe technique using Keithley electrometer, model 6514, interfaced to a PC and electrical furnace. Electrical resistance was measured at $5{ }^{\circ} \mathrm{C}$ intervals from room temperature to $300{ }^{\circ} \mathrm{C}$. Resistivity was calculated from the dimensions of the pellet and measured resistance. The thermo-emf measurement technique was reported in detail in our earlier publication [18]. The thermoemf measurements were recorded from room temperature to $400{ }^{\circ} \mathrm{C}$. Seebeck coefficient $\alpha$ was calculated from the formula:

$$
\alpha=\frac{V_{s}}{T_{2}-T_{1}}
$$

where $V_{s}$ is the thermo-emf generated and $T_{2}-T_{1}$ is the temperature difference at the hot and cold junctions which was $10^{\circ} \mathrm{C}$ in the present case. With the help of thermoelectric power studies and DC electrical conductivity measurements one can calculate the mobility of the carriers using the following equation in case of low mobility semiconductors, such as ferrites:

$$
\mu=\frac{\exp (\alpha / k / e) \sigma}{16 e} \mathrm{~cm}^{2} \cdot \mathrm{V}^{-1} \cdot \mathrm{s}^{-1}
$$

where e is the charge of electron and $\mathrm{k}$ is the Boltzmann constant.

Activation energies of the carriers were estimated from the plots of 1000/T versus $\ln \mu$ according to the relation:

$$
\mu=\mu_{o} \exp \left[\frac{-\Delta E_{\mu}}{k T}\right]
$$

\section{Results and discussion}

Typical X-ray diffraction patterns of the $\mathrm{Mg}_{0.5-\mathrm{x}} \mathrm{Cu}_{\mathrm{x}} \mathrm{Zn}_{0.5} \mathrm{Fe}_{1.9} \mathrm{O}_{4-\delta}$ shown in Fig. 1 confirm the single phase spinel structure formation. It is seen in Fig. 2 that DC electrical conductivity decreases with an increase in copper concentration in the series of the samples. The iron deficient series of the samples exhibits higher resistivity than their counterparts with stoichiometric iron content [19]. This suggests that during sintering, formation of $\mathrm{Fe}^{3+}$ to $\mathrm{Fe}^{2+}$ ions was minimized due to the shortage of iron. Materials for transformer cores or television deflection yokes should have high resistivity of the order $\sim 10^{9} \Omega \cdot \mathrm{m}$ and more, which has been attained in the present work. Yue et al. [20] reported an increase in electrical conductivity with the addition of copper, which is contrary to the results obtained in the present work.

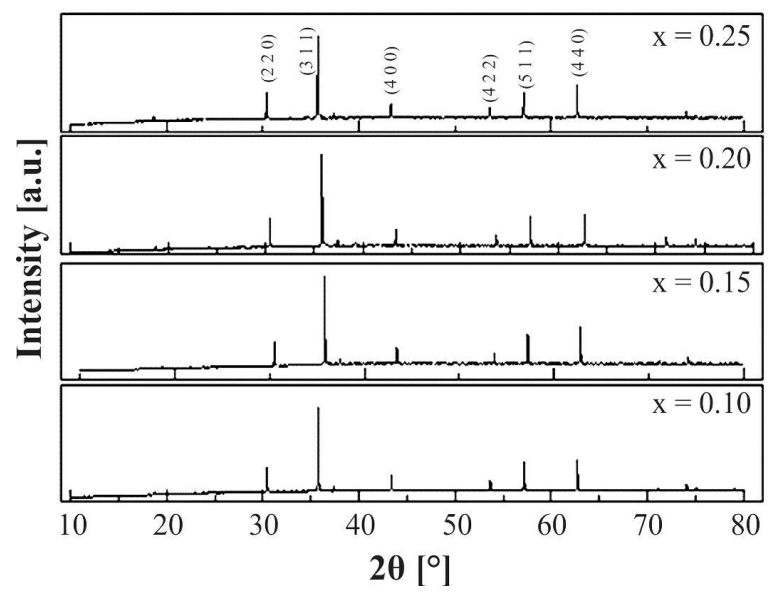

Fig. 1. X-ray diffraction patterns of $\mathrm{Mg}_{0.5-\mathrm{x}} \mathrm{Cu}_{\mathrm{x}} \mathrm{Zn}_{0.5} \mathrm{Fe}_{1.9} \mathrm{O}_{4-\delta}$.

The DC conductivity versus the reciprocal temperature for the series of the samples in the temperature range of $30{ }^{\circ} \mathrm{C}$ to $300{ }^{\circ} \mathrm{C}$ is shown in Fig. 3. The conductivity varies from $5.56 \times 10^{-9} \Omega^{-1} \cdot \mathrm{cm}^{-1}$ to $2.21 \times 10^{-5} \Omega^{-1} \cdot \mathrm{cm}^{-1}$, $5.90 \times 10^{-10} \Omega^{-1} \cdot \mathrm{cm}^{-1}$ to $2.91 \times 10^{-5} \Omega^{-1} \cdot \mathrm{cm}^{-1}$, $8.86 \times 10^{-9} \Omega^{-1} \cdot \mathrm{cm}^{-1}$ to $1.30 \times 10^{-4} \Omega^{-1} \cdot \mathrm{cm}^{-1}$ and $8.87 \times 10^{-10} \Omega^{-1} \cdot \mathrm{cm}^{-1}$ to $1.52 \times 10^{-4} \Omega^{-1} \cdot \mathrm{cm}^{-1}$ with $\mathrm{x}=0.1,0.15$, 0.2 and 0.25 , respectively. The plots are linear for all the samples with a slope change in the range of 


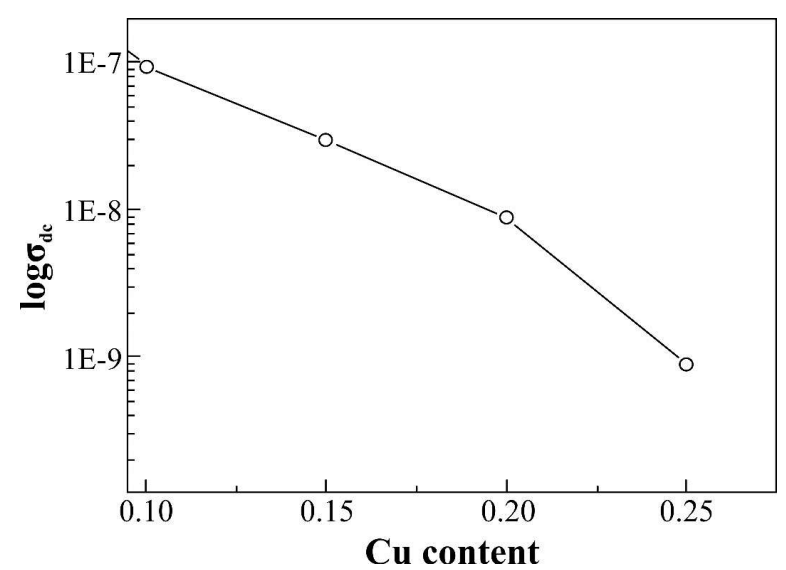

Fig. 2. Variation of DC electrical conductivity with copper concentration in $\mathrm{Mg}_{0.5-\mathrm{x}} \mathrm{Cu}_{\mathrm{x}} \mathrm{Zn}_{0.5} \mathrm{Fe}_{1.9} \mathrm{O}_{4-\delta}$.

$60{ }^{\circ} \mathrm{C}$ to $80{ }^{\circ} \mathrm{C}$, in the vicinity of magnetic Curie transition temperature $\left(\mathrm{T}_{\mathrm{c}}\right.$ from $70{ }^{\circ} \mathrm{C}$ to $\left.100{ }^{\circ} \mathrm{C}\right)$. The slope change in the graphs indicates that there are two regions of conductivity viz. low temperature (ferromagnetic) region I and high temperature (paramagnetic) region II. Similar slope change near Curie transition temperature in Arrhenius plots for DC conductivity was observed by Rezlescu et al. $[9,21]$ and it was attributed to the influence of ferromagnetic ordering on conduction mechanism. In principle, this is due to destruction of spontaneous magnetization and the change in additional charge carrier concentration in the neighborhood of Curie transition temperature. In ferrites, being magnetic semiconductors, conductivity in the low temperature region $\mathrm{I}$ is attributed to impurities and is extrinsic in nature. At high temperatures, the conductivity is intrinsic in nature and is due to electron hopping between $\mathrm{Cu}^{2+} \leftrightarrow \mathrm{Cu}^{1+}$ and $\mathrm{Fe}^{3+} \leftrightarrow \mathrm{Fe}^{2+}$ ions. From the mentioned magnitudes it is clear that the conductivity has increased by four orders of magnitude with temperature. Rezlescu et al. [9] reported an increase of DC electrical conductivity with temperature by about three orders of magnitude only in $\mathrm{Ta}_{2} \mathrm{O}_{5}$ and $\mathrm{PbO}$ added $\mathrm{MgCuZn}$ ferrites. In another work, Rezlescu et al. [21] reported an increase in DC conductivity with temperature for $\mathrm{MgCuZn}$ ferrites of about four orders of magnitude. This strong increase in conductivity with temperature was attributed mainly to the thermally activated mobility of the charge carriers, but not to thermally activated creation of these carriers. The activation energy of the charge carriers has been estimated from resistivity versus temperature plots. The results are tabulated in Table 1. The activation energies of the samples in intrinsic region are greater than those in extrinsic region.

$\begin{array}{lcclr}\text { Table } & \text { 1. Activation energies from } & \text { DC } \\ \text { conductivity studies and } & \text { mobility } & \text { of } \\ & \mathrm{Mg}_{0.5-\mathrm{x}} \mathrm{Cu}_{\mathrm{x}} \mathrm{Zn}_{0.5} \mathrm{Fe}_{1.9} \mathrm{O}_{4-\delta} . & & \end{array}$

\begin{tabular}{ccccc}
\hline & \multicolumn{2}{c}{ From DC conductivity } & \multicolumn{2}{c}{ From mobility } \\
Copper content & Region I & Region II & \multicolumn{2}{c}{ Region I Region II } \\
$\mathrm{x}$ & $\Delta \mathrm{E}_{1}[\mathrm{eV}]$ & $\Delta \mathrm{E}_{2}[\mathrm{eV}]$ & $\Delta \mathrm{E}_{1}[\mathrm{eV}]$ & $\Delta \mathrm{E}_{2}[\mathrm{eV}]$ \\
\hline \hline 0.1 & 0.45 & 0.66 & 0.23 & 0.61 \\
0.15 & 0.35 & 0.87 & 0.39 & 0.86 \\
0.2 & 0.36 & 0.92 & 0.29 & 0.81 \\
0.25 & 0.73 & 0.9 & 0.26 & 0.84 \\
\hline
\end{tabular}

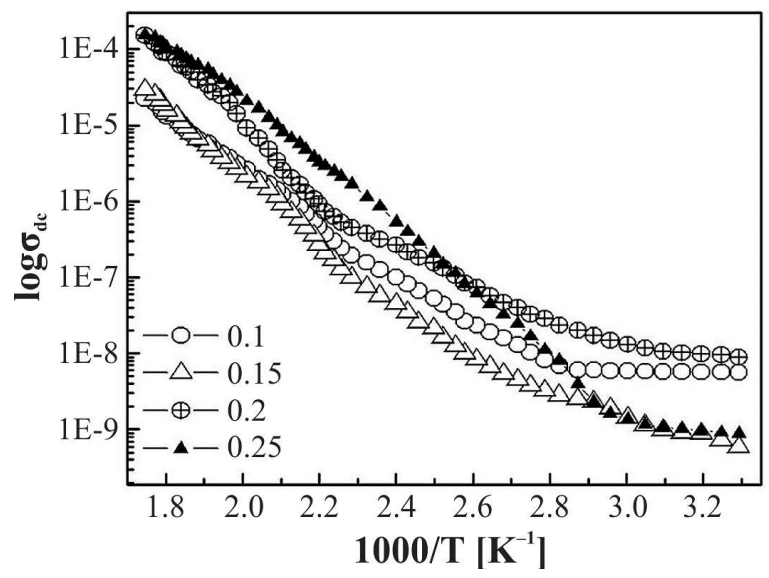

Fig. 3. Variation of DC electrical conductivity with temperature in $\mathrm{Mg}_{0.5-\mathrm{x}} \mathrm{Cu}_{\mathrm{x}} \mathrm{Zn}_{0.5} \mathrm{Fe}_{1.9} \mathrm{O}_{4-\delta}$.

The variation of Seebeck coefficient with copper concentration is shown in Fig. 4. All the samples exhibit a negative Seebeck coefficient indicating that the majority charge carriers are electrons. As the copper content increases, the magnitude of thermoelectric power decreases and reaches saturation at higher content of copper. Fig. 5 shows the variation of Seebeck coefficient with temperature for the non-stoichiometric $\mathrm{MgCuZn}$ ferrite samples. An examination of Fig. 5 reveals that there is 
a maximum of Seebeck coefficient for every composition at a temperature $\mathrm{T}_{\mathrm{s}}$. The temperature at which the maximum is obtained in Seebeck coefficient has been designated as Seebeck coefficient transition temperature $T_{\mathrm{S}}$ by Reddy et al. [22] and Ravinder [23]. It may be pointed out here that $T_{s}$ and Curie transition temperature $T_{c}$ are different from each other. The author is of the opinion that these maxima obtained are due to the charge carrier redistribution taking place at these temperatures. Repeated thermoelectric power measurements on these samples showed that there is some type of ordering taking place inside these samples.

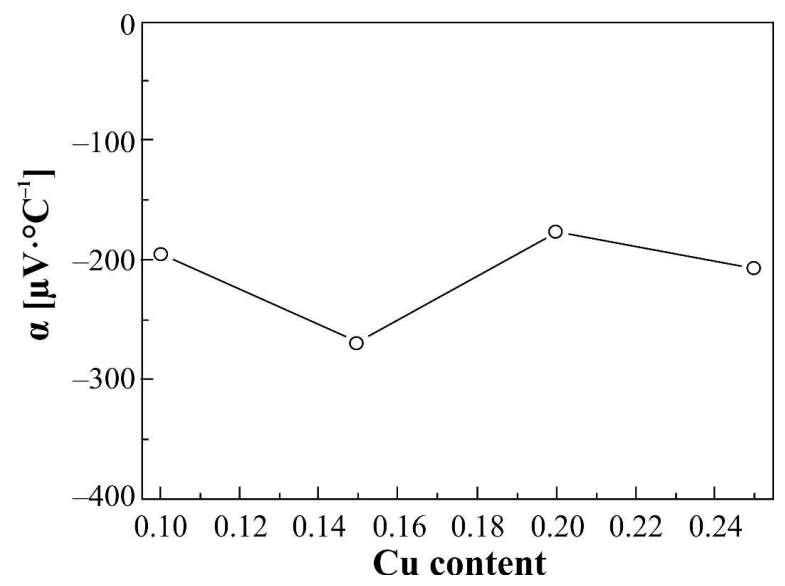

Fig. 4. Variation of Seebeck coefficient with copper concentration in iron deficient ferrite series.

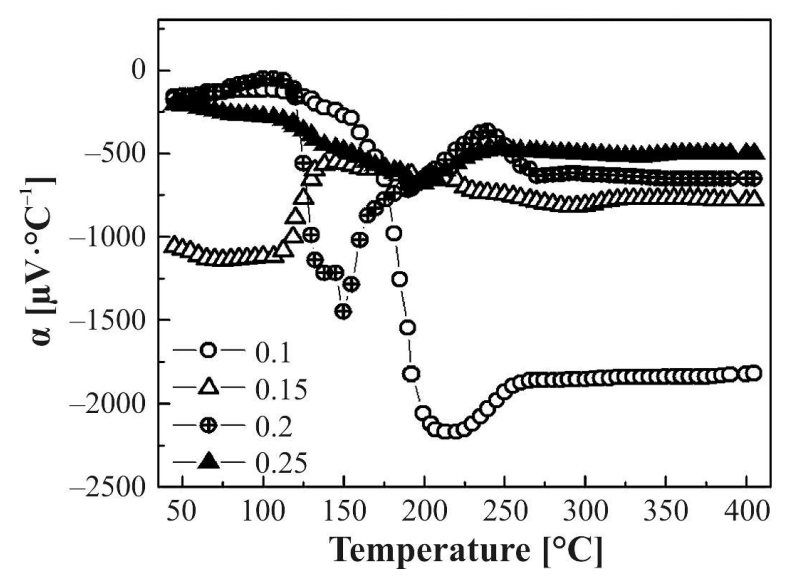

Fig. 5. Variation of Seebeck coefficient with temperature in $\mathrm{Mg}_{0.5-\mathrm{x}} \mathrm{Cu}_{\mathrm{x}} \mathrm{Zn}_{0.5} \mathrm{Fe}_{1.9} \mathrm{O}_{4-\delta}$.

The temperature dependence of charge carriers mobility for the series of the samples is shown in
Fig. 6. In the investigated temperature range all the samples exhibit two regions of conductivity. The activation energies were calculated from the plots of charge carriers mobility, $\log \mu$ versus $1 / \mathrm{T}$. The activation energies of all the samples are tabulated in Table 1.
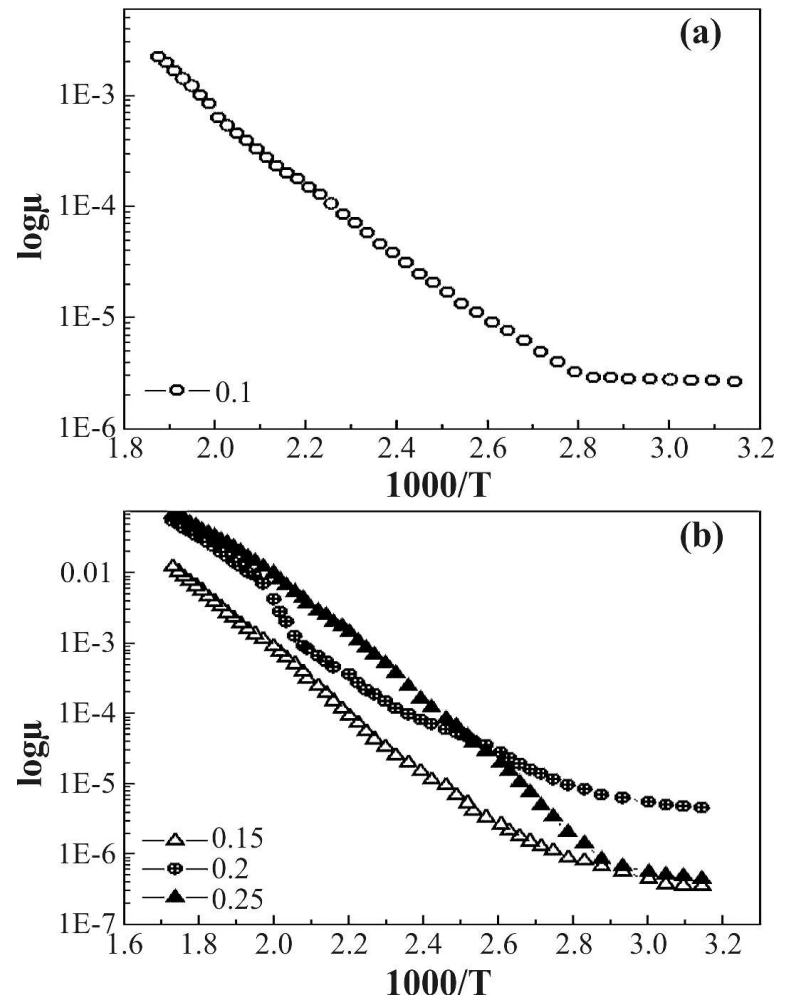

Fig. 6. Temperature dependence of mobility $\mu$ in $\mathrm{Mg}_{0.5-\mathrm{x}} \mathrm{Cu}_{\mathrm{x}} \mathrm{Zn}_{0.5} \mathrm{Fe}_{1.9} \mathrm{O}_{4-\delta}$ (a) $\mathrm{x}=0.1$ and (b) $\mathrm{x}=0.15$ to 0.25 .

The effect of ferromagnetic-paramagnetic transition could be observed even in mobility plots proportional to DC conduction. The low temperature region, where an extrinsic conduction is favoured, is shown as region I in Table 1 . In this region, the conductivity is basically due to the impurities. The activation energies $\left(\Delta \mathrm{E}_{2}\right)$ in the region II (intrinsic region) are found to be greater than those of region I. Further, the activation energies $\Delta \mathrm{E}_{2}$, estimated from DC conductivity and mobility plots are in good agreement at high $\mathrm{T}$ (temperature) region. The activation energies $\left(\Delta \mathrm{E}_{1}, \Delta \mathrm{E}_{2}\right)$ are greater than the ionization energies $\left(E_{i}=0.1 \mathrm{eV}\right)$ of the donor and acceptor atoms, thus ruling out the band 
theory of conduction. Moreover, these energies are greater than the $\mathrm{Fe}^{3+} \leftrightarrow \mathrm{Fe}^{2+}$ transition energy $\left(E_{e}=0.2 \mathrm{eV}\right)$ which rules out the possibility of electron hopping mechanism. The above discussion suggests that the conduction process may be due to polaron hopping in these mixed $\mathrm{MgCuZn}$ ferrites.

\section{Conclusions}

Nonstoichiometric $\mathrm{Mg}_{0.5-\mathrm{x}} \mathrm{Cu}_{\mathrm{x}} \mathrm{Zn}_{0.5} \mathrm{Fe}_{1.9} \mathrm{O}_{4-\delta}$ compounds with $\mathrm{x}$ varying from 0 to 0.25 have been synthesized by conventional double sintering technique. X-ray diffraction patterns confirmed the single phase spinel structure. DC electrical conductivity increased with copper concentration and temperature. Conduction in these compounds was due to electron transfer between $\mathrm{Cu}^{2+} \leftrightarrow \mathrm{Cu}^{1+}$ and $\mathrm{Fe}^{3+} \leftrightarrow \mathrm{Fe}^{2+}$. The increase in DC electrical conductivity can be attributed to thermally activated charge carriers rather than thermally created carriers. Thermoelectric studies revealed n-type conduction in the samples within the investigated temperature range. However, the studies of electrical parameters depending on temperature exhibited two regions of conduction viz., low temperature extrinsic and high temperature intrinsic regions. Activation energies of carriers in the intrinsic region are greater than in extrinsic region evaluated from DC electrical conductivity and charge carriers mobility. The Seebeck transition temperature $\mathrm{T}_{\mathrm{s}}$ does not match with the Curie transition temperature and suggests that thermal generation of electrical potential in the present ferrites is independent of magnetic ordering. Polaron hopping is suggested as the conduction mechanism for the system of ferrites. Deliberate introduction of iron deficiency has resulted in the expected decrease in conductivity. Thus, these materials are best suited as core materials for transformers and high definition television deflection yokes.

\section{References}

[1] Rahmouni H., Benali A., Cherif B., Dhahri E., Boukhobza M., Khirouni K., Sajieddine M., Physica B, 466 - 467 (2015), 31.
[2] Syed Samar Abbas, Iftikhar Hussain Gul, Shahid Ameer, Muhammad AneEs, Electron. Mater. Lett., 11 (2015), 100.

[3] Koн J.G., YU C.I., J. Korean Phys., Soc., 247 (1984), 359.

[4] Koн J.G., Kiм K.U., J. Korean Phys., Soc., 26 (1986), 540.

[5] Park J., Kim J., Cho S., J. Phys. C-Solid State Phys., 193 (1997), 79.

[6] Bhosale D.N., Choudari N.D., Sawanth S.R., BAKARE P.P., J. Magn. Magn. Mater., 173 (1997), 51.

[7] Rezlescu N., Rezlescu E., Popa P.D., Craus M.L., ReZLESCU L., J. Magn. Mag. Mater., 182 (1998), 199.

[8] Sachlarie L., Rezlescu E., Rezlescu N., Phys. Status Solidi A, 179 (2000), R1.

[9] Rezlescu N., Sachlarie L., Rezlescu E., Pope P.D., Cryst. Res. Technol., 36 (2001), 157.

[10] Qi X., Zhou J., Yue Z., Gui Z., Li L., J. Magn. Magn. Mater., 251 (2002), 316.

[11] Murbe J., Topfer J., Int. J. Appl. Ceram. Tec., 4 (2007), 415.

[12] Ladgaonkar B.P., VASAMbekar P.N., VAinGANKAR A.S., B. Mater. Sci., 23 (2000), 87.

[13] Ramana V.M., Reddy R.N., Kumar S.K.V., Phys. Res. Int., 2012 (2012), 861690.

[14] Patil S.B., Ppatil R., Chougule B.K., J. Magn. Magn. Mater, 335 (2013), 109

[15] ZAKI H.M., Physica B, 404 (2009), 3356.

[16] Radwan F.A., Ahmed M.A., Abdelatif G., $J$. Phys. Chem. Solids, 64 (2003), 2465.

[17] Kang S.-H., Chang S.-H., Yoo H.I., J. Solid State Chem., 149 (2000), 33.

[18] Madhuri W., Reddy P.M., Reddy R.M.N., KUMAR S.K.V., Int. J. Chem. Tech. Res., 6 (2014), 1771.

[19] Madhuri W., Reddy P.M., KIM I., REDDY R.M.N., KumAR S.K.V., MurThy V.R.K., Mater. Sci. Eng. B$A d v ., 178$ (2013), 843.

[20] Yue Z., Zhou J., Li L., Wang X., Gui Z., Mater. Sci. Eng. B-Adv., 86 (2001), 64.

[21] Rezlescu E., Rezlescu N., Popa P.D., ReZlescu L., Pasnicu C., Craus M.L., Mater. Res. Bull., 33 (1998), 915.

[22] Reddy D.V., Amalik M., Reddy V.P., Mater. Sci. Eng. B-Adv., 8 (1991), 295.

[23] RAVINDER D., Mater. Lett., 43 (2000), 129. 